\title{
A young child with pseudohypoaldosteronism type II by a mutation of Cullin 3
}

Shoji Tsuji ${ }^{*}$, Miyoko Yamashita ${ }^{2}$, Gen Unishi ${ }^{3}$, Reiko Takewa ${ }^{1}$, Takahisa Kimata ${ }^{1}$, Kiyoshi Isobe ${ }^{4}$, Motoko Chiga ${ }^{4}$, Shinichi Uchida ${ }^{4}$ and Kazunari Kaneko ${ }^{1}$

\begin{abstract}
Background: Pseudohypoaldosteronism type II (PHA II), also referred to as Gordon syndrome, is a rare renal tubular disease that is inherited in an autosomal manner. Though mutations in WNK1 and WNK4 partially account for this disorder, in 2012, 2 research groups showed that KLHL3 and CUL3 were the causative genes for PHA II. Here, we firstly report on the Japanese child of PHA II caused by a mutation of CUL 3.
\end{abstract}

Case presentation: The patient was a 3-year-old Japanese girl having healthy unrelated parents. She was initially observed to have hyperkalemia, hyperchloremia, metabolic acidosis, and hypertension. A close investigation led to the diagnosis of PHA II, upon which abnormal findings of laboratory examinations and hypertension were immediately normalized by administering thiazides. Genetic analysis of WNK1 and WNK4 revealed no mutations. However, analysis of the CUL3 gene of the patient showed abnormal splicing caused by the modification of exon 9 . The patient is currently 17 years old and does not exhibit hypertension or any abnormal findings on laboratory examination.

Conclusions: In this patient, CUL3 was found to play a fundamental role in the regulation of blood pressure, potassium levels, and acid-base balance.

Keywords: Pseudohypoaldosteronism, Kelch-like 3, Cullin 3, Gordon syndrome, Ubiquitination

\section{Background}

Pseudohypoaldosteronism type II (PHA II), also referred as Gordon syndrome, is a rare renal tubular disease that is inherited in an autosomal manner [1]. Because thiazides are effective in the treatment of PHA II, a genetic defect in the $\mathrm{NaCl}$ cotransporter (NCC), the target transporter of thiazides, was thought to be the cause of PHA II. However, in 2001, Wilson et al. first reported that PHA II is caused by molecular abnormalities in 2 types of WNK (WNK; With No K [lysine]) genes, i.e., WNK1 and WNK4 [2]. Yang et al. confirmed that in WNK4 knock-in mice, the NCC present in the cell membranes of the luminal surface cells of the distal tubule is excessively phosphorylated owing to a WNK4 mutation, resulting in hypertension due to abnormal electrolyte/acid-base equilibrium and increased circulating blood volume [3]. However, a considerable number

\footnotetext{
* Correspondence: tsujis@hirakata.kmu.ac.jp

'Department of Pediatrics, Kansai Medical University, 2-5-1 Shin- machi, Hirakata- shi, Osaka 573 1010, Japan

Full list of author information is available at the end of the article
}

of patients with PHA II did not exhibit any genetic defect in WNKs. Recently, genetic defects in Kelch-like 3 (KLHL3) or Cullin 3 (CUL 3) were also reported to cause PHA II [4].

Here, we present the first case, to our knowledge, of PHA II caused by a mutation of CUL3, diagnosed in a 3-year-old Japanese child.

\section{Case presentation}

Direct sequencing of DNA and mRNA and splicing assay Genomic DNA and RNA were extracted from peripheral blood lymphocytes by using a QIAamp DNA Blood Midi Kit (Qiagen, Venlo, The Netherlands) and Tempus ${ }^{\mathrm{Tw}}$ Spin RNA isolation Kit (Applied Biosystems, Foster City, CA, USA), respectively. The mRNA was reverse-transcribed with oligo(dT) primer (Omniscript RT Kit, Qiagen, Venlo, The Netherlands). A 633-bp segment of the CUL3 gene was amplified by polymerase chain reaction (PCR) (Primers: forward, 5' -TACGGAATAGAATTCCACTC-3'; reverse, 5' -CTCCATGAATGTATCCTGAC-3') from genomic DNA. A 561-bp segment (390 bp, if exon 9 was 
skipped) was amplified by PCR (Primers: forward, $5^{\prime}$-TG AGGGAGCAAGGTAAAGCTC-3'; reverse, 5' -GCACCC GGACTGTAAGATCA-3') from the cDNA. The PCR products were verified by sequencing. The ethics committee of Saiseikai Noe Hospital approved this study after consent from the patient's guardian was obtained.

\section{Case report}

The patient was a 3-year-old Japanese girl with healthy unrelated parents. Her perinatal history and past medical history were unremarkable. She was observed to have hyperkalemia, hyperchloremia, metabolic acidosis, and hypertension, when she was admitted to our hospital for the treatment of croup syndrome. An initial physical examination revealed normal growth, with a height of $95.5 \mathrm{~cm} \quad(+0.3$ standard deviation [SD]) and a body weight of $14.0 \mathrm{~kg}(+0.2 \mathrm{SD})$. The patient's development was also normal. Her body temperature was $36.4^{\circ} \mathrm{C}$. Chest auscultation revealed normal breathing sounds and a regular heart beat without murmurs. Examination of the patient's blood and blood pressure revealed hyperkalemia $(6.8 \mathrm{mEq} / \mathrm{L})$, hyperchloremia $(112 \mathrm{mEq} / \mathrm{L})$, metabolic acidosis $\left(\mathrm{pH}, 7.248 ; \mathrm{HCO}_{3}^{-}, 12.6 \mathrm{mEq} / \mathrm{L}\right)$, and hypertension $(124 / 30 \mathrm{mmHg})$. The plasma renin activity $\left(0.2 \mathrm{ng} \cdot \mathrm{ml}^{-1} \cdot \mathrm{h}^{-1}\right)$ and plasma aldosterone concentration $(25 \mathrm{pg} / \mathrm{ml})$ were also low. A closer investigation led to the diagnosis of PHA II, and abnormal findings on laboratory examinations and hypertension were promptly normalized by the administration of thiazides. Subsequent genetic analysis of WNK1 and WNK4 revealed no mutations. The CUL3 gene of the patient, her parents, and her younger brother were analyzed in 2013, based on the report by Boyden et al. [4]. Abnormal splicing caused by modification of exon 9 was detected only in the patient (Figure 1a). The skipping of exon 9 at the mRNA level was further confirmed by sequence analysis (Figure 1b) and reverse-transcription PCR (Figure 1c). Because a mutation of CUL3 and manifestation of PHA II were not observed in the family members of the patient, in this case, the mutation was considered to be de novo. The patient is currently 17 years old and is healthy, with no hypertension or abnormal findings on laboratory examinations. The most recently recorded height and body weight of the patient were $155.6 \mathrm{~cm}(-0.4 \mathrm{SD})$ and $52.6 \mathrm{~kg}(-0.1 \mathrm{SD})$, respectively.

\section{Conclusions}

Monogenic disorders such as PHA II give the opportunity to observe the clinical effects of a mutated gene. PHA II attracts special attention because its symptoms (hypertension, hyperkalemia, and acidosis) are usually caused by renal insufficiency, which is a relatively common cause of elevated blood pressure. PHA II is caused by the loss of WNK4-mediated tonic inhibition of thiazidesensitive NCC in the distal convoluted tubule, by a kinasedependent mechanism, due to loss-of-function mutations

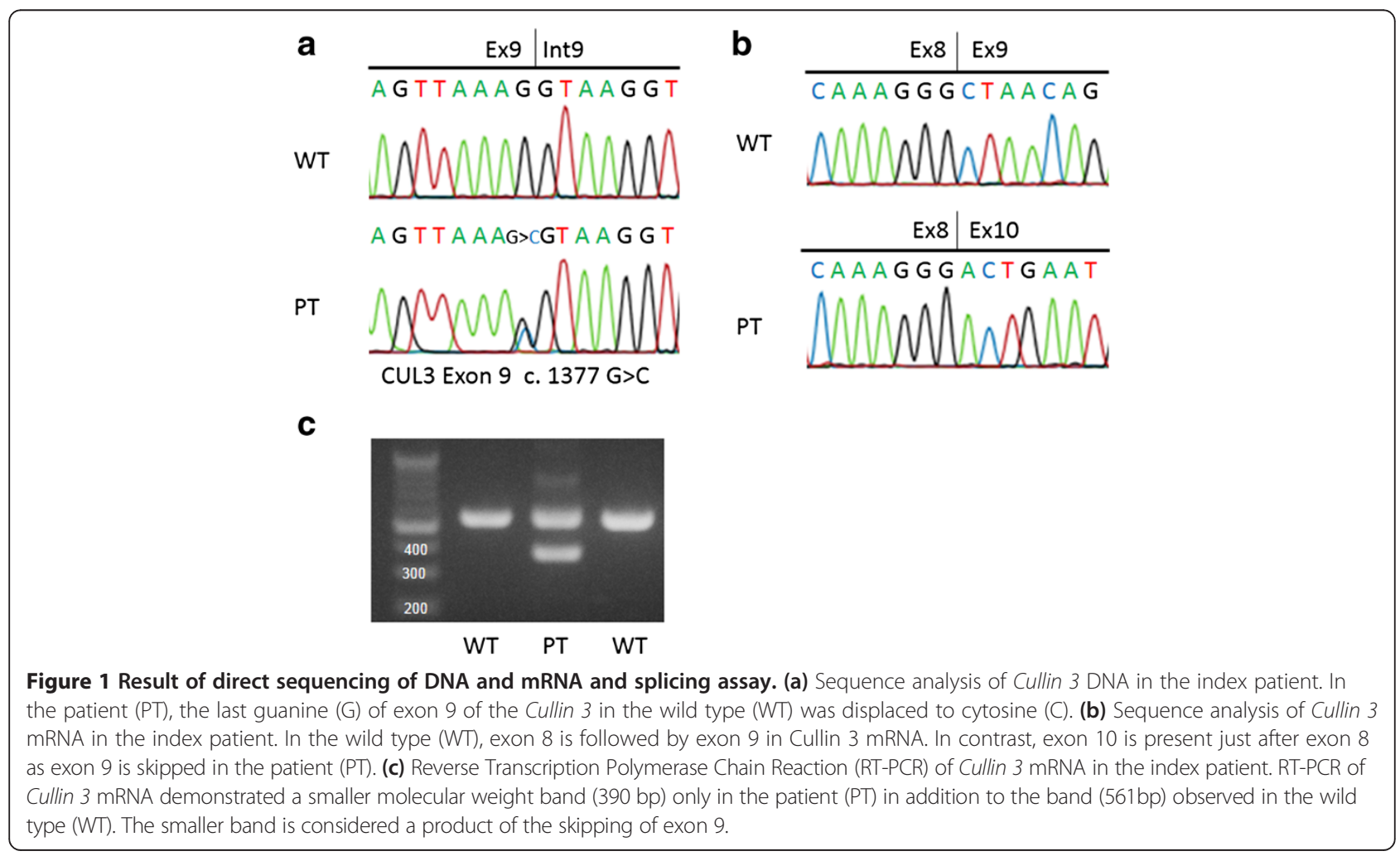


in WNK4 and gain-of-function mutations in WNK1 [2]. Though mutations in WNK1 and WNK4 are the cause of PHAII in some patients, recent successive studies have shown that KLHL3 and CUL3 are also causative genes for PHA II $[4,5]$. The CUL3 protein forms a complex with KLHL3 proteins, which then functions as a ubiquitin ligase. However, the exact role of these genes in PHAII is currently unknown. Recently, some groups reported that an interaction of KLHL3 with CUL3 and WNK4 induced WNK4 ubiquitination and reduced the WNK4 protein level, while a reduction in the interaction between KLHL3 and WNK4 attenuated the ubiquitination of WNK4, resulting in an increased level of the WNK4 protein [6-8].

The genotype-phenotype correlation in PHA II remains unknown, because only a few descriptions of PHA II in childhood have been reported [9-11]. Interestingly, Boyden et al. [4] reported that the onset of PHA II caused by CUL3 mutation is earlier and the disease is more severe than is PHA II caused by KLHL3 recessive, KLHL3 dominant, WNK4, and WNK1 mutations. In agreement with their report, the patient in the present case was diagnosed with evident hyperkalemic metabolic acidosis and hypertension at the early age of 3 years. It is noteworthy that despite the severity of the PHA II symptoms, administration of thiazides alone was sufficient to ensure normal development and growth of the patient.

This case demonstrates that KLHL3/CUL3 plays a fundamental role in the regulation of blood pressure, potassium levels, and acid-base balance.

\section{Consent}

Written informed consent was obtained from the patient for publication of this case report. A copy of the written consent is available for review by the Editor of this journal.

\section{Abbreviations \\ PHA II: Pseudohypoaldosteronism type II; NCC: NaCl cotransporter; KLHL3: Kelch-like 3; CUL 3: Cullin 3; PCR: Polymerase chain reaction.}

\section{Competing interests}

The authors declare that they have no competing interests.

\section{Authors' contributions}

ST and TK performed the literature search and drafted the manuscript. MY, GU and RT were also treating physicians for the patient. KI and MC carried out the molecular genetic studies and participated in the sequence alignmen. SU and KK conceived of the study and helped to draft the manuscript. All authors read and approved the final manuscript.

\section{Acknowledgement}

This study was partly supported by the Mami Mizutani Foundation.

\section{Author details}

${ }^{1}$ Department of Pediatrics, Kansai Medical University, 2-5-1 Shin- machi, Hirakata- shi, Osaka 573 1010, Japan. ²Department of Pediatrics, Saiseikai Noe Hospital, Osaka, Japan. ${ }^{3}$ Department of Pediatrics, Unishi Clinic, Osaka, Japan. ${ }^{4}$ Department of Nephrology, Graduate School of Medical and Dental Sciences, Tokyo Medical and Dental University, Tokyo, Japan.
Received: 11 April 2013 Accepted: 30 July 2013

Published: 31 July 2013

\section{References}

1. Gordon RD, Geddes RA, Pawsey CG, O'Halloran MW: Hypertension and severe hyperkalaemia associated with suppression of renin and aldosterone and completely reversed by dietary sodium restriction. Australas Ann Med 1970, 19(4):287-294.

2. Wilson FH, Disse-Nicodeme S, Choate KA, Ishikawa K, Nelson-Williams C, Desitter I, Gunel M, Milford DV, Lipkin GW, Achard JM, et al: Human hypertension caused by mutations in WNK kinases. Science 2001, 293(5532):1107-1112.

3. Yang SS, Morimoto T, Rai T, Chiga M, Sohara E, Ohno M, Uchida K, Lin SH, Moriguchi T, Shibuya $\mathrm{H}$, et al: Molecular pathogenesis of pseudohypoaldosteronism type II: generation and analysis of a Wnk4 (D561A/+) knockin mouse model. Cell Metab 2007, 5(5):331-344.

4. Boyden LM, Choi M, Choate KA, Nelson-Williams CJ, Farhi A, Toka HR, Tikhonova IR, Bjornson R, Mane SM, Colussi G, et al: Mutations in kelch-like 3 and cullin 3 cause hypertension and electrolyte abnormalities. Nature 2012, 482(7383):98-102.

5. Louis-Dit-Picard H, Barc J, Trujillano D, Miserey-Lenkei S, Bouatia-Naji N, Pylypenko O, Beaurain G, Bonnefond A, Sand O, Simian C, et al: KLHL3 mutations cause familial hyperkalemic hypertension by impairing ion transport in the distal nephron. Nat Genet 2012, 44(4):456-460. S451-453.

6. Wakabayashi M, Mori T, Isobe K, Sohara E, Susa K, Araki Y, Chiga M, Kikuchi E, Nomura N, Mori Y, et al: Impaired KLHL3-Mediated Ubiquitination of WNK4 Causes Human Hypertension. Cell Rep 2013, 3(3):858-868.

7. Ohta A, Schumacher FR, Mehellou Y, Johnson C, Knebel A, Macartney TJ, Wood NT, Alessi DR, Kurz T: The CUL3-KLHL3 E3 ligase complex mutated in Gordon's hypertension syndrome interacts with and ubiquitylates WNK isoforms: disease-causing mutations in KLHL3 and WNK4 disrupt interaction. Biochem J 2013, 451(1):111-122.

8. Shibata S, Zhang J, Puthumana J, Stone KL, Lifton RP: Kelch-like 3 and Cullin 3 regulate electrolyte homeostasis via ubiquitination and degradation of WNK4. Proc Natl Acad Sci USA 2013, 110(19):7838-7843.

9. Mayan H, Vered I, Mouallem M, Tzadok-Witkon M, Pauzner R, Farfel Z: Pseudohypoaldosteronism type II: marked sensitivity to thiazides, hypercalciuria, normomagnesemia, and low bone mineral density. J Clin Endocrinol Metab 2002, 87(7):3248-3254.

10. Mayan H, Munter G, Shaharabany M, Mouallem M, Pauzner R, Holtzman EJ, Farfel Z: Hypercalciuria in familial hyperkalemia and hypertension accompanies hyperkalemia and precedes hypertension: description of a large family with the Q565E WNK4 mutation. J Clin Endocrinol Metab 2004, 89(8):4025-4030.

11. Mayan H, Melnikov S, Novikov I, Holtzman EJ, Farfel Z: Familial hyperkalemia and hypertension: pathogenetic insights based on lithium clearance. J Clin Endocrinol Metab 2009, 94(8):3010-3016.

doi:10.1186/1471-2369-14-166

Cite this article as: Tsuji et al: A young child with

pseudohypoaldosteronism type II by a mutation of Cullin 3. BMC Nephrology 2013 14:166.

\section{Submit your next manuscript to BioMed Central and take full advantage of:}

- Convenient online submission

- Thorough peer review

- No space constraints or color figure charges

- Immediate publication on acceptance

- Inclusion in PubMed, CAS, Scopus and Google Scholar

- Research which is freely available for redistribution 